\title{
BMJ Open Quality Improving the accuracy of digital templating: achieving success through stakeholder management
}

\author{
Hammad Parwaiz, ${ }^{\circledR}$ Boris Aliaga Crespo, Joshua Filer, Ashley Thorpe, \\ Rosie Seatter, Dominique Suzanne Faulkner, Harvey Sandhu
}

To cite: Parwaiz H, Aliaga Crespo B, Filer J, et al. Improving the accuracy of digital templating: achieving success through stakeholder management. BMJ Open Quality 2019;8:e000378. doi:10.1136/ bmjoq-2018-000378

Received 10 March 2018 Revised 23 April 2019 Accepted 7 May 2019

\section{Check for updates}

(c) Author(s) (or their employer(s)) 2019. Re-use permitted under CC BY-NC. No commercial re-use. See rights and permissions. Published by BMJ.

Trauma and Orthopaedics, Royal United Hospitals Bath NHS Foundation Trust, Bath, UK

Correspondence to

Mr Hammad Parwaiz;

hammad.parwaiz@nhs.net

\section{ABSTRACT}

The use of a marker ball in digital templating for hip arthroplasty is a well-established method of preoperative planning and is used to overcome the inherent magnification in plain film radiographs. Our hospital policy is to place a marker ball in all anteroposterior pelvic films taken in the emergency department (ED) which have been requested for suspected neck of femur fractures. We carried out a baseline measurement followed by three Plan-Do-Study-Act cycles for all pelvic films taken in ED during July 2016, November 2016, February 2017 and November 2017. Interventions between the baseline measurement and cycle 1 were to educate the lead radiographer and publish the results in the radiology newsletter, and between cycles 1 and 2 was to run a teaching session for radiographers, display posters in the X-ray department and place an electronic prompt on the X-ray machine to alert them of the need to place a marker ball in the $X$-ray field. Cycle 3 looked to see if improvements were sustained. 16/81 (20\%) radiographs complied with the policy in our baseline measurement; $25 / 51(46 \%, p=0.002)$ in cycle $1 ; 40 / 54(74 \%, p=0.0056)$ in cycle 2 ; and $48 / 63(76 \%)$ in cycle 3 . Our quality improvement project led to large improvements in clinical practice through straightforward, small, but appropriately targeted interventions. Stakeholder management is key to successfully implementing change. The next step is to switch from the VoyantMark to the KingMark marker ball, as it has greater accuracy of templating and is also easier to place within the field of an X-ray.

\section{PROBLEN}

It is our hospital's policy to place a marker ball in all anteroposterior (AP) pelvic films taken in the emergency department (ED) which have been requested for suspected neck of femur fractures. Orthopaedic surgeons then use the TraumaCad software (TraumaCad, Petach-Tikva, Israel) to preoperatively template hip hemiarthroplasties and total hip replacements.

We have found in trauma meetings that many patients with neck of femur fractures lacked marker balls in their radiographs, or had inadequately placed marker balls. This prevented templating for procedures. Templating helps overcome some of the technical challenges of an operation, and it has medicolegal implications when patients are dissatisfied with the outcome of a procedure. This was therefore thought to be an important issue to address.

We audited the accurate and correct placement of these marker balls in all AP pelvic radiographs for suspected neck of femur fractures in ED, and implemented several interventions to try and improve clinical practice.

\section{BACKGROUND}

The use of a marker ball in digital templating for hip arthroplasty is a well-established method of preoperative planning and is used to overcome the inherent magnification in plain film radiographs caused by divergent $\mathrm{X}$-ray beams, the distance of the plate from the patient and the patient size. ${ }^{1-5}$ It enables calibration within digital templating software to help accurately predict implant sizes, select correct offset and positioning of implants, reduce leg length discrepancy and reduce intraoperative complications.

\section{MEASUREMENT}

We carried out a prospective departmental audit of all AP pelvic radiographs performed for suspected neck of femur fractures in the ED. A baseline measurement was undertaken in June 2016 followed by three PlanDo-Study-Act (PDSA) cycles; all radiographs from November 2016, February 2017 and November 2017. The hospital's picture archiving and communication system software was used to filter out only the pelvic $\mathrm{X}$-rays taken in the ED over these defined time periods.

Next, the clinical information submitted at the time of requesting the X-rays was analysed. Radiographs were excluded from analysis if the clinical information on the request did not indicate it was performed for a neck of femur fracture. Examples include 


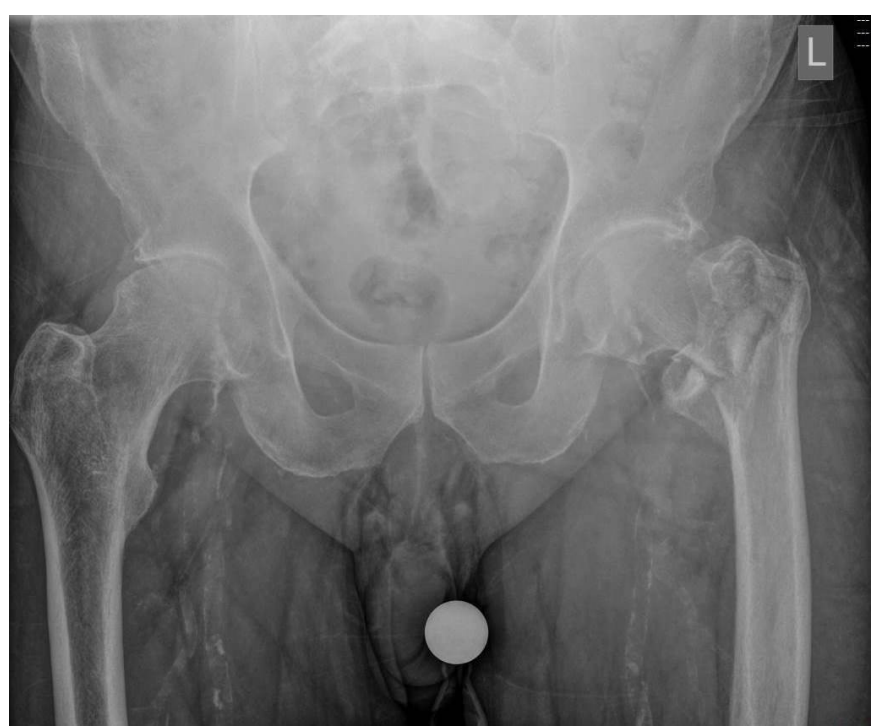

Figure 1 An example of an anteroposterior (AP) pelvic radiograph taken for a suspected neck of femur fracture in the emergency department. At the bottom of the image is an example of a well-placed marker ball.

where it was performed for suspected pubic rami fracture, infection, dislocation or paediatric cases. In order to fully adhere to hospital protocol the marker ball must be present in its entirety - this is because the templating software can only calibrate the X-rays if the entire ball is present in the image (figure 1). The exact location of the marker ball can be flexible; the vast majority are placed between the legs, however, it could also be placed lateral to the hip as long as the marker ball is at the level of the greater trochanter in the AP plane.

Data were entered manually over the course of the PDSA cycles into an Excel spreadsheet. Statistical analysis was carried out using www.graphpad.com and a Fisher's exact test was used to calculate $p$ values. A $p$ value $<0.05$ was thought to be statistically significant.

\section{DESIGN}

Interventions (table 1) between the baseline measurement and cycle 1 were to publish results of our audit in the monthly newsletter of radiographers (including the theory and importance of correct marker ball placement) and education of the lead radiographer in the ED (who could then disseminate the message to the rest of the department). Between cycles 1 and 2 we ran two practical teaching sessions for all radiographers where we presented the results of the audit and demonstrated on a volunteer how to optimally place the marker ball. In addition to this we put up posters in the radiology department within the X-ray suite to act as an aide memoire, and an electronic prompt was created on the X-ray machine so that every time the radiographer selected the option of performing a pelvic X-ray a reminder to place the marker ball was displayed on screen. We also disseminated the results of our quality improvement project at the trauma and orthopaedic audit and governance meetings. Cycle 3 was looking to see if these improvements were sustained.

\section{STRATEGY}

The status quo prior to the start of the quality improvement project was that the policy had been created but not widely known by the radiology department. It was kept in a folder within the X-ray department and difficult to access.

The success of this quality improvement project lies in stakeholder management-a phrase commonly used in business and management when discussing change and how to achieve it. We identified three main stakeholders: (1) patients, for whom a correctly sized implant is the most crucial; (2) orthopaedic surgeons, for whom templating is a routine prior to operating; and (3) radiographers who are the front-line staff members responsible for identifying the patients who need a correctly placed marker ball in their radiographs.

Of these, the radiographers were the key to affecting change. First, we had to get them engaged with the project. Why is this important? How is it relevant to them, to patients and to surgeons? What effect does an incorrectly placed or absent marker ball have on patient care? What can they do to improve this? Instead of speaking to radiographers individually, or relying solely on published reminders and posters, we engaged with the lead radiographer who was also the policy's author. Their support

Table 1 Interventions during the different PDSA cycles

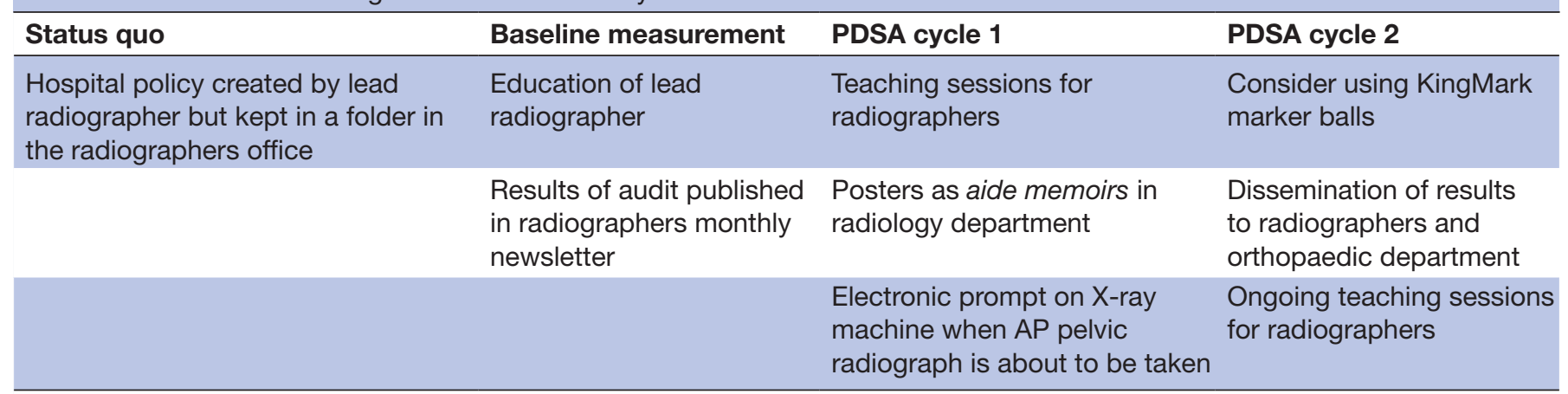

AP, anteroposterior; PDSA, Plan-Do-Study-Act. 
was gained by sitting down with them and explaining the importance and relevance of marker balls, by showing them that a published standard exists and that it was their own departmental policy, and, finally, by emphasising that improvement in practice is unachievable without their engagement.

Once the lead radiographer was on board with the project the next step was to educate the radiographers and implement changes in practices and processes. Too often in audit projects interventions fail to achieve significant change, and this can sometimes be due to lack of involvement of those people being affected by the change. The lead radiographer was consulted prior to each intervention in order to improve engagement.

The first set of interventions was to educate the lead radiographer and publish the results of the audit in the radiographers monthly newsletter. Although this did bring about an improvement in practice from $20 \%$ to $46 \%$ it was still far below what was acceptable. During the first PDSA cycle we realised that although radiographers were now aware of the policy our interventions did not do anything to address the technical difficulties they were experiencing. This led us to run two practical teaching sessions for the radiographers as part of their weekly teaching programme. We simulated the process of placing a marker ball with a volunteer and radiographers were able to discuss difficulties they had been having. These included placement of the marker ball between the legs in patients with high body mass index and how to ensure the marker ball was placed in the correct level in the AP plane. We were able to demonstrate solutions to these problems. Finally, aide memoirs and digital prompts on the X-ray machines reduced the number of radiographs where a marker ball was absent due to the radiographer forgetting the policy.

\section{RESULTS}

Ninety-two AP pelvic radiographs were performed in June 2016 and 11 were excluded as they were not performed for suspected neck of femur fracture (table 2). Sixteen of $81(20 \%)$ were compliant with the audit standard (figure 2). Seventy-four radiographs were performed in November 2016, twenty were excluded from analysis and 25/54 (46\%) were compliant $(\mathrm{p}=0.002)$. Seventy-two radiographs were performed in February 2017, 18 were excluded and 40/54 (74\%) were compliant $(\mathrm{p}=0.0056)$.

\section{Compliance With The Standard}

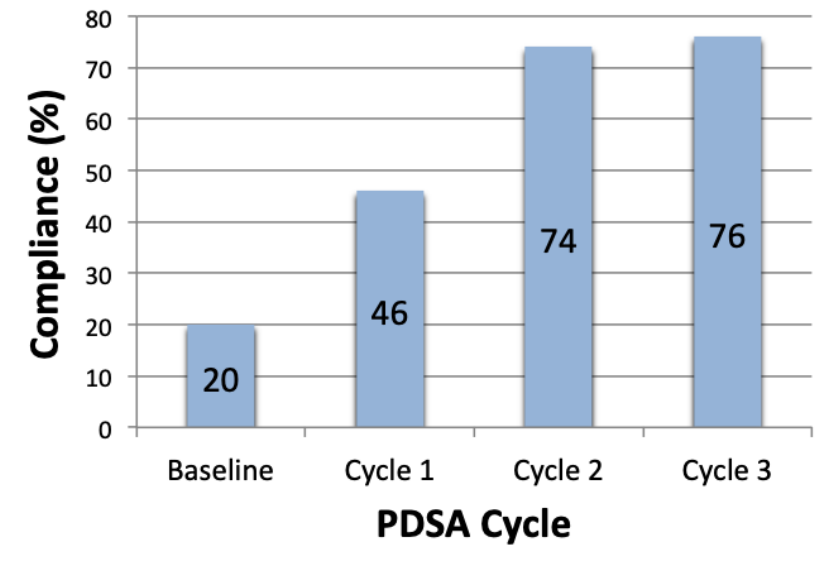

Figure 2 Compliance of pelvic radiographs with trust policy during each Plan-Do-Study-Act (PDSA) cycle.

One hundred and six radiographs were performed in November 2017, 43 were excluded and 48/63 (76\%) were compliant.

\section{LESSONS AND LIMITATIONS}

The results of our quality improvement project show that large improvements in clinical practice can be achieved by straightforward, small, but appropriately targeted interventions. There were significant improvements in practice with each PDSA cycle.

The success of this quality improvement project lies in stakeholder management and engaging with the lead radiographer. During each PDSA cycle we were in constant communication about what the results showed, brainstorming ideas of how to improve practice and working together to deliver the changes. Without the help and engagement of the lead radiographer our interventions would not have been anywhere near as effective as they were, and some ideas would not have been possible (such as the electronic prompt on the X-ray machine).

Although we were able to move from $20 \%$ to $74 \%$ compliance over the course of 8 months there is still room for further improvement. For example, we have found that occasionally ED staff were ordering hip X-rays for suspected neck of femur fractures. Although in most cases this was changed by the radiographers to an AP

Table 2 Number of pelvic radiographs that were compliant with the hospital's policy on marker balls

\begin{tabular}{llllll}
$\begin{array}{l}\text { PDSA } \\
\text { cycle }\end{array}$ & AP pelvic radiographs $(\mathbf{n})$ & Exclusions & Compliant & Non-compliant & $\begin{array}{l}\% \\
\text { Compliant }\end{array}$ \\
\hline Baseline & 92 & 11 & 16 & 65 & 20 \\
1 & 74 & 20 & 25 & 46 & 76 \\
2 & 72 & 18 & 40 & 14 & 74 \\
3 & 106 & 43 & 48 & 15 & 76 \\
\hline
\end{tabular}

AP, anteroposterior; PDSA, Plan-Do-Study-Act. 
pelvic film, there were a few that remained a hip X-ray, and therefore subsequently did not have a marker ball placed or AP pelvic X-ray performed. Both these things prevent templating. We hope to implement an algorithm-based neck of femur X-ray requesting option on our radiology requesting software that would allow the requester to make a single selection on the requesting software and the patient would then have an AP pelvic X-ray followed automatically by a chest X-ray (routine preoperative films) if a fracture was found. Second, the orthopaedic department are investigating the option of using the KingMark marker ball as a way of improving marker ball placement and templating accuracy. VoyantMark, the currently used marker ball, is a ball on a flexible gooseneck placed at the level of the greater trochanter in the AP plane. In contrast, the KingMark is a dual calibration marker ball system that has a plate that goes under the patient and a belt with marker balls that is placed around the patient. Studies have shown it to be more accurate than VoyantMark. ${ }^{6}$

Results from November 2017 show that these results are sustainable as the compliance remained similar at $76 \%$.

\section{CONCLUSION}

The results of our audit show that large improvements in clinical practice can be achieved by straightforward, small, but appropriately targeted interventions. Stakeholder management is key to successfully implementing change.

While some of our interventions are permanent (electronic prompts on the X-ray machine), others will need to be periodically repeated (such as teaching sessions for radiographers) in order to sustain long-term improvements in practice. This is largely due to staff turnover.
Acknowledgements The authors thank Stacey Hall, lead radiographer, for her help in implementing the interventions mentioned in this paper.

Contributors All authors have contributed to the project and manuscript. HP designed the project, collected data, analysed data, presented results, carried out interventions between PDSA cycles and wrote the manuscript. BAC and JF collected data, analysed data, presented results and reviewed the manuscript. AT and RS collected data, analysed data, presented results, carried out interventions between PDSA cycles and reviewed the manuscript. DSF collected data, analysed data and presented results. HS supervised the project and reviewed the manuscript.

Funding The authors have not declared a specific grant for this research from any funding agency in the public, commercial or not-for-profit sectors.

Competing interests None declared.

Patient consent for publication Not required.

Provenance and peer review Not commissioned; externally peer reviewed.

Open access This is an open access article distributed in accordance with the Creative Commons Attribution Non Commercial (CC BY-NC 4.0) license, which permits others to distribute, remix, adapt, build upon this work non-commercially, and license their derivative works on different terms, provided the original work is properly cited, appropriate credit is given, any changes made indicated, and the use is non-commercial. See: http://creativecommons.org/licenses/by-nc/4.0/.

\section{REFERENCES}

1. Eggli S, Pisan M, Muller ME. The value of preoperative planning for total hip arthroplasty. Journal of Bone and Joint Surgery 1998;80B:382-90.

2. Kwok IHY, Pallett SJC, Massa E, et al. Pre-operative digital templating in cemented hip hemiarthroplasty for neck of femur fractures. Injury 2016;47:733-6.

3. Lakstein D, Bachar I, Debi R, et al. Radiographic templating of total hip arthroplasty for femoral neck fractures. Int Orthop 2017;41:831-6.

4. Shin JK, Son SM, Kim TW, et al. Accuracy and reliability of preoperative On-screen templating using digital radiographs for total hip arthroplasty. Hip Pelvis 2016;28:201-7.

5. Steinberg EL, Shasha N, Menahem A, et al. Preoperative planning of total hip replacement using the TraumaCad ${ }^{\mathrm{TM}}$ system. Arch Orthop Trauma Surg 2010;130:1429-32.

6. King RJ, Makrides P, Gill JA, et al. A novel method of accurately calculating the radiological magnification of the hip. J Bone Joint Surg Br 2009;91:1217-22. 